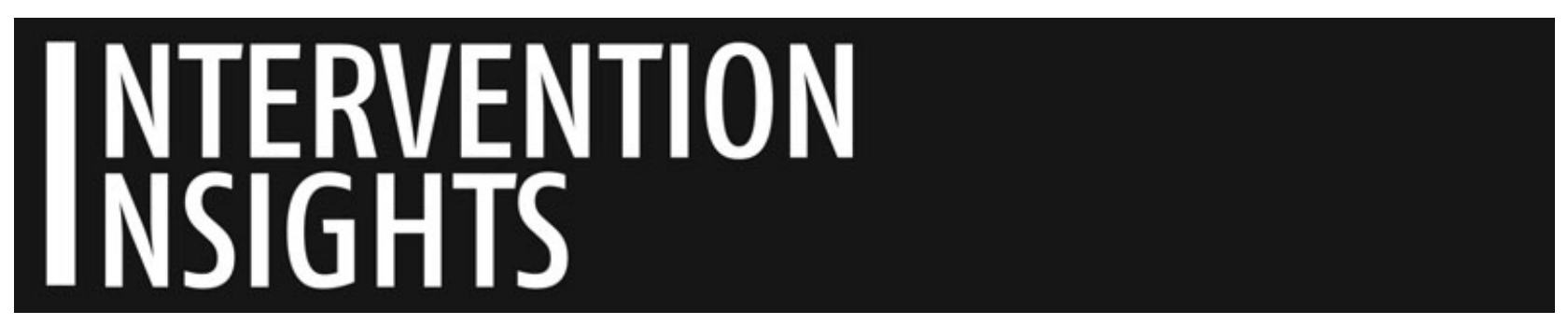

\title{
Termination of therapy: what can clinicians do to maximise gains?
}

\begin{abstract}
The progress a patient makes during therapy should be enriched and heightened by a positive treatment ending. When termination issues are ignored or mishandled, the whole of therapy is jeopardized.
\end{abstract}

- Kramer (1990, p. xv)

Although there has been a significant emphasis in the clinical literature on therapeutic engagement and process, there has been surprisingly little focus on how to conclude therapy in a way that maximises gains.

Preparing for termination of therapy can be extremely important when working with most clinical populations. The clinician may have played a significant role, often at a time when a patient is most distressed, and it is likely that there could be some sadness or concern in relation to this potential loss of support. Indeed, Ryle and Kerr (1) noted that '... as termination is approached, the absence of anxiety and disappointment would suggest that the reality of the end is not yet felt by the patient ...' (p. 113).

Although a sense of loss may be experienced by most clinical populations when concluding therapy, additional care may need to be taken with patients who have experienced difficult attachment histories, particularly when this has included significant loss or abandonment. Sensitive management is also required when difficulties remain at the conclusion of therapy and the patient does not feel 'cured', and particularly when the sense of hope that may have been present at the commencement of therapy is replaced by sadness or anger. Termination with individuals who have experienced these difficulties is arguably the most important part of therapy, and if managed well, can give the patient a sense that endings need not be traumatic or inevitably involve a profound sense of loss. Mathews (2) noted that 'Despite its attendant complications, therapist-initiated terminations, like other endings, provide potentially important grist for the growth mill' (p. 34).

Having said this, it is important that the clinician does not assume the patient's response to the termination of therapy. It can be surprising when some patients for whom therapy appears to have been extremely important do not seem overly concerned by termination, whereas others appear significantly more affected than the clinician may have anticipated. As Kramer (3) succinctly noted 'To each patient, termination means something different ...' (p. 63). Perhaps the easiest solution to this potential difficulty is for the clinician to simply ask what concluding therapy means to each patient and to provide time for discussion about this.

\section{Types of 'Termination'}

Service considerations and the clinician's theoretical model are likely to dictate at least partially how and when therapy terminates. However, given that patients are likely to have different needs, Kramer (3) suggested that the clinician may want to consider different types of endings. These can include reducing the frequency of sessions, taking 'breaks' when the person is asymptomatic or having an 'open door' policy once therapy has officially ended. It is likely that for a number of people-even following a seemingly successful conclusion of therapy-some concern will remain regarding the possibility of recurrence or exacerbation of the presenting difficulties.
Therefore, it is not uncommon to receive phone calls or occasional visits from people who have officially finished therapy. It appears important that the clinician remains available, whenever possible, in these situations, even if the main focus is on encouraging the person's engagement with another service. For many individuals who are sensitised to abandonment and who manage the ambivalence of intimacy by avoidance, drop-out may be their way of anticipating and taking control of termination.

\section{Therapist challenges}

It should be noted that part of the difficulty in concluding therapy comes not only from concern experienced by the patient, but also from concerns experienced by the therapist. Davis (4) summarised some potential difficulties that therapists can experience regarding termination including: '.. the avoidance of uncomfortable affect, under-assertiveness ..., lack of clarity about possible reasons or criteria for termination, discomfort with interpersonal change, or a fear of precipitating some harm by discontinuing treatment too soon' (p. 78). Patients will have beliefs relating to gains that have been made, what may need further work, and loss of the clinician. However, clinicians are also likely to evaluate how the patient has progressed, reflect on the intervention he or she has provided and also consider the impact that finishing with the patient has on him/herself. As Kottler (5) noted of finishing therapy, 'The clinician may feel guilt, failure, disappointment, sadness, pride, apprehension, hope, jealousy and relief-all at once. And there is the constant cycle of growing immensely fond 
of people and then turning them loose' (p. 94). It is therefore important for the clinician to have time to acknowledge his or her own thoughts and feelings regarding the end of therapy with each patient. Although workplace demands may mean that there are constantly people waiting to be seen, it is important that the clinician takes time to reflect on termination and if possible, not have another appointment immediately after a final session with a patient.

\section{Timing of termination}

One of the challenges of termination can be identifying when it should occur, with Freud (6) having noted, 'Is there such a thing as a natural end to an analysis?' (p. 219). From a psychodynamic psychotherapy viewpoint, Kramer (3) defined key termination criteria as therapist's intuition, symptom relief, improved intrapsychic functioning, resolution of transference and interpreting dreams relating to termination. However, Kramer also concluded, ‘. . criteria for termination vary greatly. Even within the same school of thought, there are differences in opinion. I propose that the whole idea of criteria may be irrelevant and possibly damaging to therapy itself. It is likely that all abstract and theoretical discussions of termination criteria are of little value and perhaps actually contribute to practitioners' and patients' confusions about the process of ending treatment' (p. 59). Regarding more brief therapy, Henry et al. (7) stated 'The purpose of time-limited therapy is not to cure patients of all their problems; rather it is to help individuals develop skills to manage and overcome further problems and challenges' (p. 54). Similarly, Milton Erickson offered the somewhat reassuring statement 'Therapy is often a matter of tipping the first domino' (8) (p. 41). Again, however, we recognise that therapeutic model, financial considerations and the setting in which the clinician practices may have considerable influence. It is also notable that with the best planning and intentions, therapy may also end when the patient makes the decision unilaterally by failing to turn up.

\section{Planning for termination}

It is necessary to begin planning for termination of therapy at the commencement of therapy. When first discussing the process of therapy with an individual, a framework for the expected timeline of the therapy needs to be introduced with a clear expectation of how the therapy will terminate. There is huge variability in this process, from the open-ended model of analytic therapies to the highly structured nature of certain managed care approaches to reimbursement, that for example only allow six sessions for a particular indication. The process needs to be structured to manage expectations, to set goals and to provide a framework for dealing with crisis, non-response or recurrence.

\section{The goodbye letter}

Narrative and cognitive analytic therapies describe the use of therapeutic letters at the conclusion of therapy $(1,9)$. These letters are used to document issues that have arisen during the course of therapy and to summarise and reflect. They can also describe the shared formulation reached during therapy, progress made, and how this could be maintained or strengthened. Importantly, the narrative therapy literature also uses letters to allow therapists to reflect on, and where appropriate, disclose their own experience of therapy with the patient. This can include describing challenges that they have experienced, what he or she has enjoyed about work with that particular patient and what the clinician has learnt from the experience him/herself (10).

Discussion about such a letter can also be an opportunity for the therapist to receive feedback about key messages the patient has retained, what the patient has not found particularly helpful, and what was most valuable about the therapy. Discussion at this point can also be a final opportunity to correct any

misunderstandings, examine any areas the therapist has neglected to include and also allow the therapist to model that sadness, disappointment and anxiety, in addition to hope and optimism, may be appropriate.

\section{Summary and guidelines}

As mentioned earlier, how termination is undertaken likely to be, at least in part, dictated by the service in which the clinician practices and by his or her therapeutic model. However, the following recommendations may be useful regardless of therapeutic orientation. Specifically:
- Termination should be on the agenda early in therapy. This can be challenging, particularly when a patient initially presents as hopeless or distressed, or for whom there are already concerns about engagement. The cognitive analytic therapy literature (1) advises discussing termination in the first session, suggesting that this allows for planning of the end of therapy by both clinician and patient, and encourages the full utilisation of all sessions. Similarly, Davis (4) advised 'Communications about termination at the beginning and throughout therapy are essential to effective management of this important task' (p. 47).

- Taking time to set appropriate goals early can prevent aimless sessions that may paradoxically be more difficult to end. Time can also be spent ensuring that goals are realistic within the time period that the clinician is able to offer.

- Regular reviews can focus therapy and allow for ongoing discussion regarding termination.

- Although it can be tempting for the clinician to minimise the importance of termination of therapy to the patient to reduce any anxiety, it is important that sufficient time is given to allow both the clinician and patient to express concerns and optimism regarding the future post-therapy.

- Although core beliefs, schema, reciprocal roles or transference can be acted out throughout the course of therapy, the termination phase can lead to an acute presentation of key concepts such as abandonment, rejection, perceived failure, entitlement and perfectionism, both on the part of the patient and the clinician. However, this can provide an extremely valuable final opportunity for the clinician to work in the 'here and now' with issues that could continue to affect the patient, and also impact on the clinician's own practice.

- Termination can offer the clinician and patient time for a valuable 'all cards on the table' exercise, where both can voice what they think could have gone better, and what they have been happy with. Encouraging honest feedback can be one of the most valuable opportunities for a clinician to request advice from patients who could potentially improve his or her clinical skills.

- Narrative therapy encourages the clinician to reflect on what he or she has gained from contact with the patient during the sessions. This can be particularly 
important for patients who may minimise their own contributions to therapy and place excessive credit for success on the therapist.

- Although use of letters summarising therapy can be challenging to complete in a busy practice, these can be very valuable and both reinforce gains made and serve as a reminder to clients of key concepts long after therapy as finished.

- Rather than providing generic positive feedback, or overly optimistic platitudes about future success for the patient, it can be important for clinicians to be precise about what they believe has improved during the course of therapy, while also asking the patient to specify their own experience of change.

- The clinician should ensure that he or she takes the time to hear realistic concerns expressed by the patient regarding termination.

- Relapse prevention work should be revisited towards the end of therapy to ensure that key strategies have been retained by the patient.

\section{Conclusions}

In conclusion, the termination of therapy can be challenging for both patient and clinician. As noted in the opening quote,

termination can be a significant event, offering dangers relating to undermining previous work done, while also presenting opportunities for consolidating gains made. However, if sufficient time is taken, if managed sensitively and if used to consolidate and strengthen gains made, termination can be one of the most powerful phases of therapy.

\section{Craig A. Macneil' ${ }^{1}$, Melissa K. Hasty ${ }^{1}$, Philippe Conus ${ }^{1,2}$ Michael Berk ${ }^{1,3,4}$}

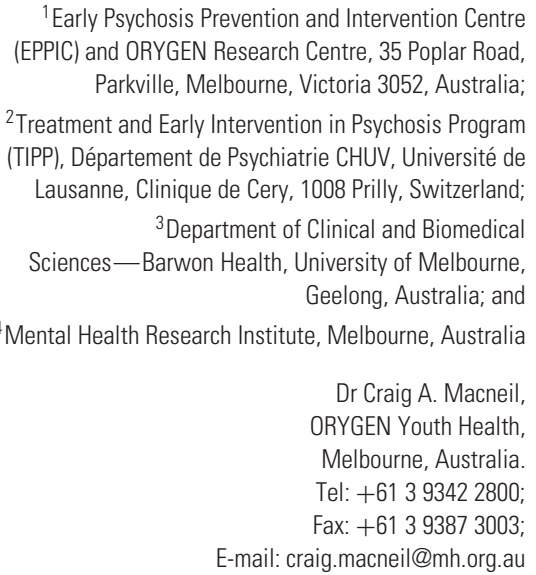

Acta Neuropsychiatrica 2010: 22: 43-45

(c) 2010 John Wiley \& Sons A/S

DOI: $10.1111 / \mathrm{j} .1601-5215.2009 .00443 . x$

\section{References}

1. Ryle A, Kerr I. Introducing cognitive analytic therapy: principles and practice. West Sussex: John Wiley \& Sons, Ltd., 2002.

2. Mathews B. Terminating therapy: implications for the private practitioner. Psychother Priv Pract 1989;7:29-39.

3. Kramer SA. Positive endings in psychotherapy: bringing meaningful closure to therapeutic relationships. San Francisco, CA: Jossey-Bass Publishers, 1990.

4. DAvIS DD. Terminating therapy. A professionals guide to ending on a positive note. Hoboken, NJ: John Wiley \& Sons, Inc., 2008.

5. Kottler JA. On being a therapist, 3rd edn. San Francisco, CA: Jossey-Bass Publishers, 2003.

6. Hillman J. The myth of analysis: three essays in archetypal psychology. IL: Northwestern University Press, 1998.

7. Henry L, Edwards J, Jackson H, Hulbert C, McGorry, P. Cognitively Oriented Psychotherapy for First-Episode Psychosis (COPE). Victoria: EPPIC, 2002.

8. O'Connell B. Solution-focused therapy. London: Sage Publications, 2005.

9. Freedman J, Combs G. Narrative therapy: the social construction of preferred realities. New York: Norton, 1996.

10. O'KeEfe P, Berk M. Writing letters to patients. Acta Neuropsychiatr 2009; 21: 314-316. 